\title{
Prevalence and characterization of methicillin- resistant Staphylococcus aureus isolates from healthy university student athletes
}

\author{
Anna E Champion', Thomas A Goodwin², P Gunnar Brolinson², Stephen R Werre', M Renee Prater ${ }^{1,2}$
} and Thomas J Inzana $a^{1,3^{*}}$

\begin{abstract}
Background: The prevalence of methicillin-resistant Staphylococcus aureus (MRSA) has been increasing in the general population, and there is concern that close or physical contact, such as in professional and collegiate sports, may increase spread of MRSA. We sought to determine the prevalence of MRSA colonization of male and female athletes from 9 different sports at a major, Division I University during a 12-week period, and determine the USA and SCCmec type from select isolates.

Methods: Swabs for culture of MRSA were obtained from nasal, axillary, and inguinal sites from healthy, asymptomatic student athletes and support staff each week for 12 weeks. Select MRSA isolates were typed by pulsed field gel electrophoresis (PFGE), and the genes encoding for MecA, cassette chromosome recombinase (Ccr), and several toxins were determined by multiplex polymerase chain reaction (PCR). Discrepant results were clarified by multi-locus sequence typing (MLST) and spa typing.

Results: Thirty-five percent (78/223) of test subjects were positive for MRSA during the study period, resulting in isolation of 139 MRSA isolates. However, 47\% (37/78) of MRSA-positive participants carried MRSA in axillary or inguinal sites, but not in the anterior nares. There was significant correlation between MRSA carriage and participation in wrestling (76\%, 19/25; adjusted odds ratio 29.7, 95\% Cl 5.8-151.5) and baseball (44\%, 17/39; adjusted odds ratio 4.4, 95\% Cl 1.1 - 17.4), compared with a staff prevalence of $18.1 \%$ (4/22), but other factors were not examined. Multiplex PCR analysis indicated that of the 32 isolates examined 26 could be typed, and all of these carried the SCCmec type IV cassette. PFGE typing identified USA types 300,400, 500, 700, and 800. However, one isolate was not a known USA type, but was identified as a novel ST951 by MLST, and as spa type t216. Of the strains typed from the same individual, there was consistency, but also variation and alternation of the SCCmec and spa types isolated from individual subjects. Various staphylococcal toxin genes were identified in 31 of the 32 isolates analyzed.

Conclusions: Colonization by MRSA was greater in some student athletes than the average carriage rate for the general population, and only 53\% of MRSA carriers were identified by nasal cultures. Carriage of MRSA clones on the same individual and transmission to contacts could vary over time, indicating colonization can be a dynamic process that may be difficult to control.
\end{abstract}

Keywords: MRSA, Methicillin-resistant, Staphylococcus aureus, Student athletes, SCCmec type, Spa type, MLST, Pulsed field gel electrophoresis, Multilocus PCR

\footnotetext{
* Correspondence: tinzana@vt.edu

${ }^{1}$ Virginia-Maryland Regional College of Veterinary Medicine, Blacksburg, VA, USA

${ }^{3}$ Virginia Tech Carilion School of Medicine, Virginia Polytechnic Institute and State University, Life Sciences 1, 970 Washington St. SW, Blacksburg, VA 24061, USA

Full list of author information is available at the end of the article
} 


\section{Background}

Methicillin-resistant Staphylococcus aureus (MRSA) was first recognized in the early 1960's as the causative agent of some hospital-acquired (HA) infections, and now accounts for the majority of HA infections in the United States [1,2]. Community-acquired (CA)-MRSA can be defined as a methicillin-resistant isolate acquired outside of a hospital setting, was acquired within 2 days of hospital admission, or was isolated from a person who has not been hospitalized within 2 years prior to the date of MRSA isolation [3]. CA-MRSA was first recognized in the early 1990's in Western Australia [4]. By the late 1990's CA-MRSA [3] had spread worldwide [5], gaining prominence after the death of 4 young children from necrotizing pneumonia who did not have underlying risk factors [6]. CA-MRSA has since become recognized as a major public health concern in the United States, and there is concern it might reach epidemic proportions [7]. Hypervirulence and/or high rates of transmission are trademarks of CA-MRSA, with the ability to cause disease in otherwise healthy individuals. CA-MRSA can cause infections similar to that of HA-MRSA, such as soft tissue and skin infections, often with abscess formation. However, serious life threatening systemic infections, such as necrotizing pneumonia, necrotizing fasciitis, bloodstream infection, and septic shock can also result from CA-MRSA $[8,9]$.

There have been multiple reports of CA-MRSA infections on college and high school campuses, with a concentration of cases occurring among student athletes [10]. Epidemiological studies have shown that coaching staff and athletes in contact and non-contact sports, as well as spectators, have been implicated as carriers during outbreaks of CA-MRSA [11]. The mecA gene, which encodes for the modified penicillin-binding protein $2 \mathrm{a}$ (PBP2a) [12], is primarily responsible for methicillin resistance in $S$. aureus. The $m e c A$ gene is carried on a mobile genetic element, named the staphylococcal cassette chromosome mec (SCCmec), which has integrated into the $S$. aureus genome [13]. At this time, at least 11 types of SCCmec elements (I-XI) have been identified [14]. These SCCmec elements share similar characteristics, and contain a $c c r$ (cassette chromosome recombinase), which is responsible for site-specific insertion and excision of SSCmec into the S. aureus genome at the 3' end of the open reading frame (orf X) [15]. SCCmec elements are differentiated based on combinations of the types of mec and $c c r$ genes that have been identified thus far. The two smallest SCCmec elements are SCCmec IV and SCCmec V, which are primarily associated with CA-MRSA. The majority of CA-MRSA strains carry a type 2 ccr-class B mec complex in a SCCmec IV cassette [16]. The SCCmec IV cassette is small compared to the SCCmec cassettes found in HA-MRSA strains, and often lacks other antibiotic resistance genes besides $\operatorname{mec} A$ [16]. Okuma et al. [16] hypothesized that the smaller size SCCmec IV requires a lower cost of fitness than the larger, multi-drug resistant SCCmec (normally found in HA isolates), and as a result would favor acquisition and retention of the cassette into the genome. CA-MRSA strains are also more likely to carry toxin genes, including Panton-Valentine leukotoxin ( $p v l$ ), staphylococcal enterotoxins (sea, seb, sec, sed, and see), toxic shock staphylococcal toxin-1 (tst), and leukocidins (lukF-PV and lukS-PV [lukFS-PV]), than HA-MRSA strains. However, not all CA-MRSA strains produce these toxins [17-19].

Chambers et al. [20] specifically examined the rates of nasal carriage of MRSA among asymptomatic athletes and coaching staff of infected individuals, and concluded there was little benefit to mass screening. However, those authors suggested that surveillance of multiple sites might improve the detection rates for colonization of asymptomatic carriers due to the presence of distinct flora on other areas of the body. The aim of this study was to assess the prevalence of MRSA colonization among athletes attending a major Division I University and their support staff, characterize the molecular basis of methicillin resistance in some of these isolates, and determine if isolates were exchanged between athletes. The persistence or variance of a genotyped strain on a subject and within a sport throughout the course of a 12-week study was determined. We hypothesized that participants in high contact sports or who experienced repeated exposure to commonly used fomites (e.g. sports equipment) would have a higher incidence of CA-MRSA than participants in low contact sports and who used primarily their own equipment. The results of this research should be noted by coaches, athletic trainers, and sports physicians to make them aware of the higher potential for MRSA infection by some athletes, but to also note that MRSA carriage is not restricted to the nares and does not necessarily result in infection.

\section{Methods}

\section{Specimen collection}

Two hundred twenty-three participants were included in the study, which included predominately college-age athletes, but also 22 of the training and medical sports staff. Athletes and staff with a prior history of MRSA infection (within 12 months) were included and documented as such. Two students reported previous MRSA infections and the course of treatment was noted. All participants were active members of a sports team during the 20072008 winter/spring season unless otherwise stated and healthy with no indication of skin or other infections at the start of the study. Whether they lived on or off campus was noted, but not ethnicity, pets, smoking, and whether roommates or family had a previous history of 
MRSA infection. Every member of the team was swabbed except for twenty-one athletes (25.8\% wrestling (WTK), $26.3 \%$ baseball (BSB), $12.5 \%$ men's track (MTK), 3.2\% women's Lacrosse (LAX) who refused consent and were excluded. There were no incentives (see Limitations and future work). Specimens from the anterior nares, axillary, and inguinal regions were obtained for culture from each individual with the BBL CultureSwab ${ }^{\text {mix }}$ (BD Diagnostics, Sparks, $\mathrm{MD})$ at exactly weekly intervals for 12 weeks, as per the manufacturer's protocol, in the physician's Sports Medicine office suite. After collection, specimens were immediately transported to the laboratory for inoculation onto culture medium. This study was approved by the university Institutional Review Board and was carried out exactly as described in the approved protocol (number 2007/041).

\section{MRSA isolation}

Swabs of the anterior nares were streaked directly onto $\mathrm{BBL}^{\mathrm{Tw}}$ CHROMagar ${ }^{\mathrm{mw}}$ MRSA plates (BD Diagnostics, Sparks, $\mathrm{MD})$, which allowed positive confirmation if colonies were mauve at $24 \mathrm{~h}$ and presumptive identification if colonies became mauve by $48 \mathrm{~h}$. At $48 \mathrm{~h}$, mauve colonies were collected and confirmed as $S$. aureus by the BBL Coagulase Plasma test (BD Diagnostics). At the time of study CHROMagar $^{\text {Tix }}$ MRSA plates were approved for identification of MRSA only from nasal swabs. Therefore, standard MRSA isolation techniques were used for axillary and inguinal swabs. Both methods have a greater than $95 \%$ specificity for identification of MRSA-positive colonies [21]. Swabs sampled from the axillary and inguinal regions were streaked onto BBL Mannitol Salt Agar (BD Diagnostics) and incubated for 24-48 h. Yellow colonies were subcultured onto Trypticase $^{\text {Tw }}$ Soy Agar with 5\% Sheep Blood (TSA II) (BD Diagnostics) and incubated at $35^{\circ} \mathrm{C}$ for $18-24 \mathrm{~h}$. Following incubation on TSA II, colonies were Gram-stained and tested for coagulase production. Coagulase-positive strains were then tested for resistance to oxacillin using BBL Oxacillin Screen Agar (BD Diagnostics), and oxacillin-resistant colonies were recorded as MRSA. The overall procedure for identifying MRSA from each type of culture swab is shown in Figure 1. When necessary, confirmation of oxacillin resistance was confirmed by minimum inhibitory

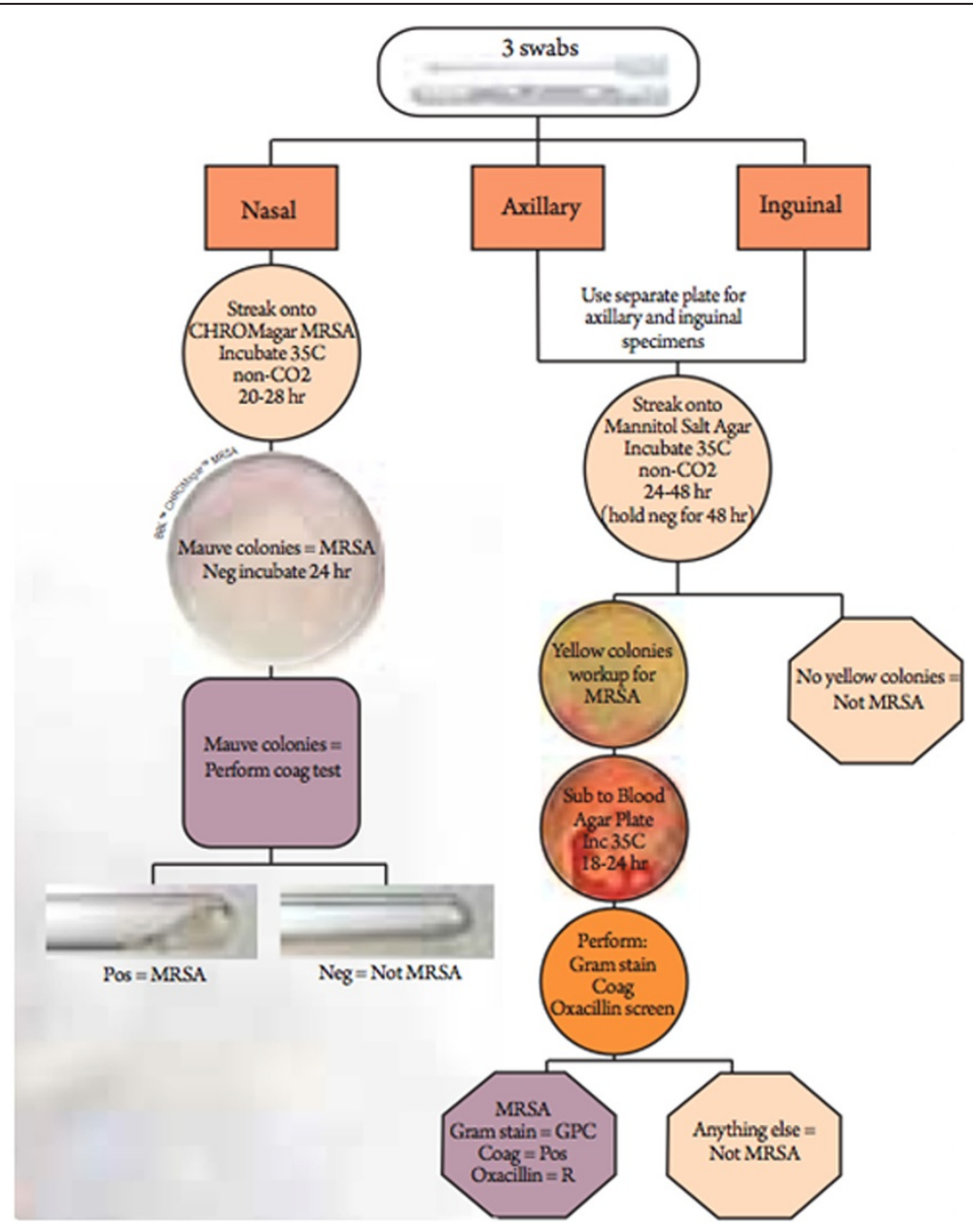

Figure 1 Flowchart of isolate collection and MRSA isolation. 
concentration (Sensititre, TREK Diagnostic Systems, Cleveland, $\mathrm{OH}$ ), following approved protocols (M07-A8 and M100-S22) described by the Clinical Laboratory Standards Institute (Wayne, PA). For the purposes of this study, an individual who was "colonized" was defined as having 1-2 positive MRSA cultures over the 12 -week period, while an individual who had 3 or more positive cultures during that time period was defined as a "persistent carrier" [22].

\section{Strain classification using pulse-field gel electrophoresis (PFGE)}

PFGE was performed as described by McDougal et al. [23]. Briefly, MRSA isolates were suspended in PIV buffer $(1 \mathrm{M} \mathrm{NaCl}, 10 \mathrm{mM}$ Tris- $\mathrm{HCl}, \mathrm{pH} 7.5)$ to an optical density of 1.2-1.7 at $580 \mathrm{~nm}$, and embedded in $1.6 \%$ InCert agarose gel plugs (Lonza Rockland, Inc. Rockland, $\mathrm{ME})$. The cells were lysed in the plugs with lysozyme, and the cell debris removed before enzyme digestion with SmaI. The plugs were loaded onto a $1.2 \%$ agarose gel for PFGE using a CHEF-DR II apparatus (Bio-Rad, Hercules, CA). Electrophoresis parameters were $200 \mathrm{~V}$ $(6 \mathrm{~V} / \mathrm{cm})$, temperature $4^{\circ} \mathrm{C}$, initial time $5 \mathrm{sec}$, final time $40 \mathrm{sec}$, and the total run time was $20 \mathrm{hr}$. Gels were stained using $1.5 \mu \mathrm{l} / \mathrm{ml}$ of ethidium bromide. PFGE types were assigned by matching the banding patterns of isolates to those of the CHEF lambda DNA standard (Bio-Rad, 1703635) and to control strains of known USA type. Photographed gel profiles were analyzed with BioNumerics software (Applied Maths, Kortrijk, Belgium) in comparison to established profiles of USA type strains [24].

\section{SCCmec genotyping by PCR}

Approximately 5 bacterial colonies from a single clone were suspended in $100 \mu \mathrm{l}$ of water and heated at $100^{\circ} \mathrm{C}$ for 10 minutes to extract DNA. Multiplex PCR was performed as described by Zhang et al. for SCCmec and ccr typing [25]. Eight pairs of primers were used for SCCmec types I, II, III, IVa, IVb, IVc, IVd, and V, 2 pairs of primers for the mec gene, 4 primers for types 1,2 , or 3 $c c r$, and 1 primer pair for type $5 \mathrm{ccr}$. The primers used were exactly those previously described for amplification of these genes [25]. Amplification was initiated at $94^{\circ} \mathrm{C}$ for $5 \mathrm{~min}$ followed by 10 cycles of $94^{\circ} \mathrm{C}$ for $45 \mathrm{~s}, 65^{\circ} \mathrm{C}$ for $45 \mathrm{~s}, 72^{\circ} \mathrm{C}$ for $1.5 \mathrm{~min}$, and then 25 cycles of $94^{\circ} \mathrm{C}$ for $45 \mathrm{~s}, 55^{\circ} \mathrm{C}$ for $45 \mathrm{~s}, 72^{\circ} \mathrm{C}$ for $1.5 \mathrm{~min}$, and a final extension at $72^{\circ} \mathrm{C}$ for $10 \mathrm{~min}$. PCR reactions were performed in triplicate to reduce error.

\section{Toxin gene identification by PCR}

DNA was extracted from approximately 5 bacterial colonies, as described above, and $1 \mu \mathrm{l}$ of extract containing the DNA was added to each reaction mix. Multiplex PCR was performed as described [26]. Briefly, primer pairs designed for amplification of sea, seb, sec, sed, see, $f e m A, m e c A$, eta, etb, and tst, were separated into 2 sets of primer mixes, with $f e m A$ included in mix $\mathrm{B}$, as described [26]. A simplex reaction containing lukFS-PV primers (F-5 TTACACAGTTAAAGAA and R-5' AATGCAATTGATG) (20 pmol) was used for detection of the $p v l$ toxin gene. The amplification protocol was initiated at $94^{\circ} \mathrm{C}$ for $5 \mathrm{~min}$ followed by 35 cycles of $94^{\circ} \mathrm{C}$ for $2 \mathrm{~min}, 57^{\circ} \mathrm{C}$ for $2 \mathrm{~min}$, $72^{\circ} \mathrm{C}$ for $1 \mathrm{~min}$, and a final extension at $72^{\circ} \mathrm{C}$ for $10 \mathrm{~min}$. The PCR reactions were performed in duplicate.

\section{Multi-locus sequence typing (MLST)}

An isolate that was untypable by multiplex PCR and PFGE was subjected to MLST using previously published primers and conditions [27]. MLST sequence types were determined through the website, www.mlst.net. A USA 300, $p v l+$ strain was used as a control.

\section{spa typing}

To confirm results determined by PFGE and SCCmec typing, the polymorphic $\mathrm{X}$ region of the spa gene was amplified using primers spa1095F (5'-AGACGATCC TTCGGTGAGC-3') and spa1517R (5'-GCTTTTGCA ATGTCATTTACTG-3') and sequenced as described previously [28]. The sequences were analyzed using Ridom StaphType software version 2.2.1 (Ridom $\mathrm{GmbH}$, Germany).

\section{Statistical analyses}

Data were summarized as the median (range) for age, class year, and number of roommates while frequency tables were generated for the categorical variables that included sport, residence (off campus vs. dorms), previous soft tissue infection, previous MRSA diagnosis, and MRSA carrier status (the primary outcome). The statistical null hypothesis was: there is no association between MRSA carrier status and the hypothesized risk factors for playing a specific sport or other category. The results were tested using univariate and multivariate analyses. For univariate analyses, associations between carrier status and the demographics of age, class year, and number of roommates were tested using the Wilcoxon 2 Sample Test. Associations between carrier status and each type of sport, gender, residence, and previous soft tissue infection were tested using the Pearson Chi-Square Test. The association between carrier status and a previous MRSA diagnosis was tested using the Fisher's Exact Test. Data on race were not available for statistical analysis. For multivariate analyses, all the hypothesized risk factors (excluding gender) were subjected to stepwise logistic regression modeling to select possible predictors for carrier status. Gender was excluded from multivariate analyses because it was perfectly correlated with the 
sport. All analyses were performed using SAS version 9.1; Cary, NC, USA.

\section{Results}

\section{Prevalence of MRSA carriage}

Two-hundred twenty-three subjects were enrolled in the study. Overall, 139 total positive cultures were obtained from 78 subjects over the 12-week study period. The prevalence for MRSA carriage was 34.9\% (78 out of 223 subjects) (Table 1). Sixty-two of the positive cultures were obtained from the anterior nares, 19 from axillary swabs, and 58 from the inguinal region. Of the 78 positive subjects, 19 harbored MRSA in more than one site, and 59 were positive at only one site (22 nasal, 6 axillary, 31 inguinal). Thus, 37 of the 78 MRSA-positive participants $(47 \%)$ were not colonized in the anterior nares (data not shown; some isolates are presented in Table 2).

Univariate analysis indicated there was a significant correlation between MRSA colonization, the sport (76.0\% [19/25] for men's wrestling vs. 43.6\% [17/39] for men's baseball vs. $57.1 \%$ [4/7] for women's tennis vs. 42.1\% [8/19] for women's softball vs 30.0\% [3/10] for men's tennis vs. $37.9 \%$ [11/29] for men's track vs. $25.0 \%$ [7/28] for women's track vs. $12.5 \%$ [4/32] for women's lacrosse; $\mathrm{p}<0.0001)$, and gender $(48.5 \%$ [50/103] for men vs. $24.5 \%$ [24/98] for women; $\mathrm{p}=0.0004)$, as well as a previous history of skin or soft-tissue infection $(72.2 \%$ [13/18] for yes vs. 35.4\% [62/175] for no; $p=0.0023$ ). There was no statistically significant association with residence status $(36.1 \%$ [26/72] for dorms vs $42.6 \%$ [49/ 115 ] for off-campus; $p=0.377$ ), class year (median was 2 [second year on campus] for both colonized and noncolonized individuals, range 1-4 and $1-5$, respectively; $\mathrm{p}=0.709$ ), age (median was 20 years for individuals colonized with MRSA vs. 19.0 for individuals not colonized, range for both groups $18-23 ; \mathrm{p}=0.312$ ), or number of roommates (median was 2.5 for individuals colonized with MRSA vs. 2.0 for individuals not colonized, range for both groups $0-9 ; \mathrm{p}=0.116$ ). Only 2 participants has a previous history of a MRSA infection, which would not support a meaningful conclusion.

Multivariate analysis of the factors analyzed indicated that the sport was the only predictor of MRSA colonization. Table 1 also shows sport-specific classification using the medical staff as the baseline. Compared with a staff prevalence of $18.1 \%(4 / 22)$, the only athletes for which colonization with MSRA was significantly more common than to the comparator group (medical staff) were in men's wrestling $(76 \%, 19 / 25$; adjusted odds ratio $29.7,95 \%$ CI 5.8 - 151.5; $\mathrm{p}<0.0001)$ and men's baseball $(43.6 \%, 17 / 39$; adjusted odds ratio 4.4, CI $1.1-17.4 ; \mathrm{p}=0.036)$ (Table 1 ). When individual sports were compared with the total number of athletes colonized with MRSA, men's wrestling proved to have the highest percentage of colonized participants (19 out of 25; 76.0\%) (Table 1, Figure 2). Women's basketball had the lowest number of colonized and persistent carrier participants, followed closely by men's tennis. There were no persistent carrier participants on the women's lacrosse team (Figure 2). In other sports the sport colonization rates varied from $8.3-57.1 \%$, and the overall colonization rates were 1.25 to $21.0 \%$ (Table 1). However, these numbers reflect the percent of participants who had at least one MRSA isolate recovered during the 12-week study. Persistent carriers (subjects with 3 or more MRSA isolates during the 12-week test period), were observed only in men's wrestling and baseball (see above). The other sport's persistent carrier rates dropped to $2.5-13.6 \%$, which was not significantly different from the comparator group (Medical Staff, data not shown). The low number of strains carried by more than one person was likely affected by the fact that, on average, approximately one third of participants were unavailable at the weekly collection time. Therefore, 12 samples from each site were not able to be obtained from every participant during the study. Weeks

Table 1 Prevalence of MRSA by sport in comparison to medical staff

\begin{tabular}{lllll}
\hline Sport played & $\begin{array}{l}\text { \#MRSA carriers/total \# } \\
\text { athletes enrolled per sport }\end{array}$ & $\begin{array}{l}\text { percentage colonized per sport/ } \\
\text { percentage colonized overall }\end{array}$ & $\begin{array}{l}\text { Adjusted odds ratio } \\
(\mathbf{9 5 \% )} \text { Cl) }\end{array}$ & $\begin{array}{l}\text { P-value based on } \\
\text { comparison to staff }\end{array}$ \\
\hline Men's wrestling & $19 / 25$ & $76.0 / 23.8$ & $29.7(5.8-151.5)$ & $<0.0001$ \\
Men's baseball & $17 / 39 \mathrm{avb}$ & $43.6 / 21.0$ & $4.4(1.1-17.4)$ & 0.036 \\
Women's tennis & $4 / 7$ & $57.1 / 4.9$ & $4.2(0.6)-29.4)$ & 0.143 \\
Women's softball & $8 / 19$ & $42.1 / 9.9$ & $4.1(0.9-19.0)$ & 0.069 \\
Men's tennis & $3 / 10$ & $30.0 / 3.7$ & $3.8(0.6-22.0)$ & 0.140 \\
Men's track & $11 / 29$ & $37.9 / 13.6$ & $3.5(0.8-14.6)$ & 0.091 \\
Women's basketball & $1 / 12$ & $8.3 / 1.25$ & $\mathrm{~N} / \mathrm{A}^{\mathrm{b}}$ & $\mathrm{N} / \mathrm{A}^{\mathrm{b}}$ \\
Women's track & $7 / 28$ & $25.0 / 4.9$ & $2.0(0.4-8.9)$ & 0.371 \\
Women's lacrosse & $4 / 32$ & $12.5 / 4.9$ & $0.8(0.2-4.2)$ & 0.832 \\
Staff & $4 / 22$ & $18.1 / 1.8$ & $1($ reference group) & -
\end{tabular}

${ }^{a}$ Homer and Lemeshow Goodness-of-Fit Chi-Square for the multivariate model was $0.000(P=1.000)$ indicating a good fit.

bxcluded from the multivariate model because only 1 participant has a positive result for MRSA. 
Table 2 Molecular characteristics of 32 MRSA isolates carried by winter/spring athletes at a Division I University

\begin{tabular}{|c|c|c|c|c|c|c|c|c|}
\hline \multicolumn{2}{|c|}{ Isolate source } & \multirow[b]{2}{*}{ Week } & \multirow[b]{2}{*}{ Sampling site ${ }^{d}$} & \multirow[t]{2}{*}{ PFGE type ${ }^{a}$} & \multirow[t]{2}{*}{ spa type } & \multirow[b]{2}{*}{ ccr-mec } & \multicolumn{2}{|c|}{ Multiplex typing $\mathrm{PCR}^{\mathrm{b}}$} \\
\hline Sport $^{c}$ & Participant \# & & & & & & SCCmec Type & Toxin gene profile \\
\hline WR & 35 & 5 & I & USA700 & $n / d$ & $2-B$ & $\mathrm{IVb}$ & eta, lukFS-PV \\
\hline WR & 41 & 6 & । & USA700 & $n / d$ & $2-B$ & $\mathrm{IVb}$ & eta, lukFS-PV \\
\hline WR & 41 & 10 & 1 & USA700 & $n / d$ & $2-B$ & $\mathrm{IVb}$ & eta, lukFS-PV \\
\hline WR & 47 & 3 & A & Unknown ${ }^{e}$ & t216 & $2-B$ & Undetermined $^{f}$ & seb, sed, etb, tst, lukFS-PV \\
\hline WR & 52 & 5 & I & USA500 & tXAA & $2-B$ & $\mathrm{IVb}$ & sea, see, eta, lukFS-PV \\
\hline WR & 52 & 6 & I & USA700 & $n / d$ & $2-B$ & $\mathrm{IVb}$ & eta \\
\hline WR & 55 & 2 & N & USA300 & t008 & $2-B$ & IVd & none detected \\
\hline WR & 55 & 3 & $\mathrm{~N}$ & USA300 & t008 & $2-B$ & IV & lukFS-PV \\
\hline WR & 55 & 3 & A & USA300 & t008 & $2-B$ & IVa & etb, tst, lukFS-PV \\
\hline WR & 55 & 5 & $\mathrm{~N}$ & USA300 & t008 & $2-B$ & IV & lukFS-PV \\
\hline WR & 55 & 7 & । & USA300 & t008 & $2-B$ & IV & sec, etb, tst, lukFS-PV \\
\hline WR & 55 & 7 & $\mathrm{~N}$ & USA300 & t008 & $2-B$ & Undetermined $^{f}$ & eta, lukFS-PV \\
\hline WR & 55 & 8 & $\mathrm{~N}$ & USA300 & $n / d$ & $2-B$ & IV & etb, tst, lukFS-PV \\
\hline WR & 55 & 8 & 1 & USA300 & t008 & $2-B$ & IV & etb, tst, lukFS-PV \\
\hline WR & 57 & 1 & $\mathrm{~N}$ & USA400 & $\mathrm{t} 128$ & $2-B$ & IVa & sea, see \\
\hline WR & 57 & 3 & N & USA400 & $\mathrm{t} 128$ & $2-B$ & IVa & seb, sec, sed, see, etb, tst, lukFS-PV \\
\hline WR & 57 & 5 & N & USA400 & $n / d$ & $2-B$ & IVa & seb, sec, see, etb, tst, lukFS-PV \\
\hline WR & 57 & 8 & $\mathrm{~N}$ & USA400 & $\mathrm{t} 128$ & $2-B$ & IVa & eta, lukFS-PV \\
\hline WR & 57 & 9 & N & USA400 & $n / d$ & $2-B$ & IVa & seb, see, eta \\
\hline WR & 57 & 12 & $\mathrm{~N}$ & USA400 & $n / d$ & $2-B$ & IVa & seb, see, lukFS-PV \\
\hline WR & 65 & 12 & $\mathrm{~N}$ & USA800 & t088 & $2-B$ & Undetermined $^{f}$ & tst \\
\hline WR & 81 & 6 & N & USA800 & t002 & $2-B$ & Undetermined $^{f}$ & lukFS-PV \\
\hline SB & 86 & 1 & I & USA400 & t008 & $2-B$ & IVa & sea, see, eta, lukFS-PV \\
\hline SB & 90 & 1 & N & USA800 & $n / d$ & $2-B$ & IVd & etb, lukFS-PV \\
\hline SB & 90 & 5 & I & USA800 & t002 & $2-B$ & Undetermined $^{f}$ & etb, lukFS-PV \\
\hline SB & 90 & 5 & N & USA800 & $\mathrm{t} 128$ & $2-B$ & Undetermined $^{f}$ & lukFS-PV \\
\hline SB & 90 & 7 & $\mathrm{~N}$ & USA800 & $n / d$ & $2-B$ & IVd & eta, lukFS-PV \\
\hline SB & 90 & 8 & $\mathrm{~N}$ & USA800 & t002 & $2-B$ & IVa & lukFS-PV \\
\hline BSB & 108 & 5 & A & USA300 & txAB & $2-B$ & IV & etb, lukFS-PV \\
\hline BSB & 108 & 11 & I & USA300 & t334 & $2-B$ & IVd & lukFS-PV \\
\hline BSB & 111 & 1 & । & USA300 & t008 & $2-B$ & $\mathrm{IVa}$ & sec, etb, tst, lukFS-PV \\
\hline WBB & 234 & 1 & I & USA400 & $n / d$ & $2-B$ & IVa & seb, see, eta \\
\hline
\end{tabular}

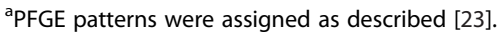

${ }^{\mathrm{b}}$ Multiplex PCR for SCCmec and ccr typing was carried out as described [25], and for toxin gene identification [26].

'WR: Wrestling, SB: Softball, BSB: Baseball, WBB: Women's Basketball.

${ }^{\mathrm{d}}$ Sampling site: I, inguinal; A, axillary; N, nasal.

eUnknown PFGE pattern; MLST was identified as ST951.

${ }^{f}$ Failed to specifically subtype using primers for SCCmec I-IV.

with no collection data for a participant were not included in statistical analyses. Medical staff screening yielded 4 MRSA colonized participants among a total of 22 participants (18.2\%).

\section{PFGE analysis}

Of the 139 MRSA isolates collected, 32 confirmed MRSA isolates were selected for further characterization based on repeated participant colonization or based on participation in a sport with a high persistent carriage rate. The 32 MRSA isolates further tested were from 13 individuals and consisted of five different pulse-field types (PFT): USA300, USA400, USA500, USA700, and USA800 [23] (Table 2 and Figure 3). Of the 13 participants represented in Table 2 from whom MRSA isolates were characterized, 6 were colonized with USA300/400 


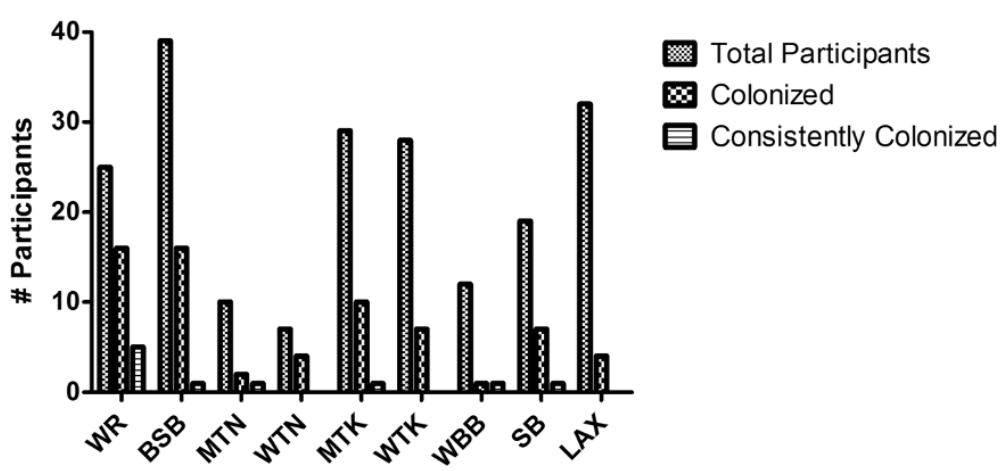

Figure 2 Prevalence of MRSA colonization in athletes by sport. Men's wrestling had the highest percentage of MRSA carriers (19 out of 25 participants or 76.0\%). In other sports, 8.3-57.14\% of participants carried MRSA. Athletes participating in men's wrestling $(p=0.0023)$ and men's baseball $(p=0.036)$ were statistically significant for colonization with CA-MSRA. WR: wrestling, BSB: baseball, MTN: men's tennis, WTN: women's tennis, WBB: women's basketball, SB: softball LAX: women's lacrosse.

MRSA types. Of interest was that in this population 3 of the participants were colonized with USA700 MRSA isolates (one participant was also colonized with USA500 [Figure 3]) and 3 were colonized with USA800 MRSA isolates. One isolate (a wrestler's axillary isolate; 47A-3) was not classified into any of the known USA strain designations after digestion with SmaI and PFGE.

\section{SCCmec typing}

All of the isolates tested that could clearly be typed harbored SCCmec IV (Table 2). Of the 26 isolates confirmed to harbor SCCmec IV, subtyping was successful in 20 of these. Eleven were subtype IVa, 5 were subtype
$\mathrm{IVb}$, and 4 were subtype IVd. None of the isolates harbored SCCmec IVc. Of the isolates tested in this study (Table 2), six isolates (47A-3, $55 \mathrm{~N}-7,65 \mathrm{~N}-12,81 \mathrm{~N}-6$, 90I-5, and $90 \mathrm{~N} 5)$ were unable to be classified into a known SCCmec type using primers for SCCmec I-IV, and IVa-IVd, which is not uncommon [29]. Since the completion of this study several new SCCmec types have been discovered indicating the vast genetic diversity of the SCCmec, and it is possible these undetermined isolates could be a newer type not tested for. Isolates 90I-5 and $90 \mathrm{~N}-5$ were from the same baseball participant on the same collection date, but from different sites. All isolates tested were of $c c r-m e c$ type 2-B.

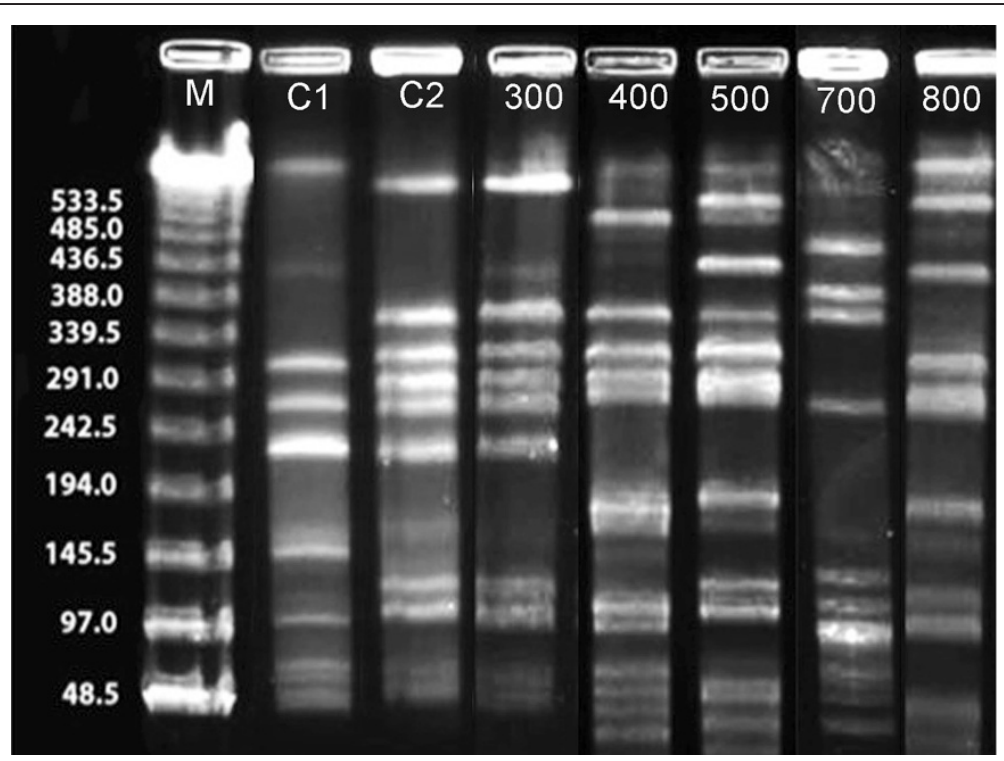

Figure 3 Examples of Smal-PFGE banding patterns of MRSA isolates. The PFGE-tenable isolates were categorized into 5 USA types. Lanes 500 and 700, representing USA types USA500 and USA700, were isolated from the inguinal site of the same individual 1 week apart. Lanes: M, molecular size standards; C1, control MRSA strain BAA1685, type USA200; C2, control MRSA strain BAA1556, type USA300; 300, type USA300 isolate; 400, type USA400 isolate; 500, type USA 500 isolate; 700, type USA700 isolate; 800, type USA800 isolate. 


\section{MLST and spa typing}

The unclassified isolate 47A-3 was subjected to MLST typing and was identified as a clonal type of ST951. This same isolate was also found to be spa type t216. There are no previously published MRSA strains with these two profiles. Isolate 47A-3 could be a novel S. aureus strain, and may be the reason it did not fall into a previous classification type by PFGE or SCCmec typing. Isolates that exhibited clonal variation within a subject over the 12-week period or those isolates that were untypeable by either previous method were also selected for spa typing. Twenty-one isolates were tested, which were classified into $8 \mathrm{spa}$ types. Three subjects encompassing 9 of the isolates tested were spa type t008. Six of these isolates were from subject 55 and all were of PFGE type USA300. The spa types t128 and t002 were each found in the same softball subject, but from two different sites obtained on the same date (nares and inguinal); both strains were USA800 and were of unclear SCCmec type. The spa types t128 and t002 were also isolated from two different wrestlers. The spa types $\mathrm{t} 216, \mathrm{t} 088, \mathrm{t} 334, \mathrm{txAB}$, and txAA (the latter two are novel spa types) were recovered only once, but may have been represented in isolates not tested.

\section{Detection of toxin genes}

Nine staphylococcal toxin genes were detected among the 32 MRSA isolates analyzed (Table 2). The most common were lukFS-PV (Panton-Valentine leukocidin) (81\%; 26 of 32 isolates; $19 \%$ of isolates tested positive for only lukFS-PV), and the exfoliative toxins eta $(34 \%, 11$ of 32 isolates), and $e t b$ (34\%; 11 of 32$)$. Other toxin genes detected included the enterotoxin genes see ( $25 \%, 8$ of 32 ), sea (9\%, 3 of 32), seb (19\%, 6 of 32), sec (13\%, 4 of 32$)$, and sed $(6 \%, 2$ of 32$)$. Nine isolates carried the toxic shock toxin gene tst (28\%). A single nasal isolate (55 $\mathrm{N}-2$ ) carried by participant 55 had no toxin genes, but other isolates recovered from participant 55 over the 12week study were positive for the presence of multiple toxin genes.

\section{Isolate variation over time}

Isolates from several participants who were repeatedly colonized with MRSA were characterized in order to identify isolate changes over 12 weeks. Wrestling participant 55 carried one clone: USA300 of spa type t0008 for 8 weeks. Wrestling participant 57 was colonized with MRSA for 6 of 12 weeks; all isolates were USA400 clones, spa type t128. Five isolates from participant 90 were USA800 isolates, but of those tested, two different spa types were identified: t002 and t128. Similarly, two isolates from baseball participant 108 were both of USA300, but of distinct spa types. In addition, two different USA types were identified from wrestler \#52,
1 week apart, and both were from the inguinal region (Table 2). Throughout the study, isolates were recovered from these participants that had varying toxin gene profiles. Of those harboring isolates with toxin genes, the most prevalent throughout were seb, see, and lukFS-PV.

Isolate characteristics were also examined between participants within the same sport. Two transiently colonized wrestling participants had the same USA700 clone containing eta and lukFS-PV toxin genes. Participant 55 was colonized for 8 weeks with a USA300 clone, but different toxin genes were identified in the isolates. Of the 22 MRSA isolates from wrestlers that were genetically characterized, there were 5 different clones (USA300, $400,500,700$, and 800 ) and one unidentifiable isolate type. Participant 57 was continuously colonized with a USA400 clone, while participants 65 and 81 were transiently colonized with a USA800 isolate with varying toxin genes (data not shown). The isolates analyzed from baseball participants were all from a USA300 clone that carried varying toxin genes. From our study, it is unclear whether this was one USA300 clone that gained or lost toxin genes throughout the 12 weeks or separate clones of USA300. The genes lukFS-PV were the only toxin genes consistently identified from all the isolates from baseball participants. However, because up to one-third of the participants were not available for culture each week, the persistence or variation of isolates from a given athlete were incomplete.

\section{Discussion}

During the past 15 years CA-MRSA isolates have been recovered from otherwise healthy individuals outside of the hospital setting. Infections due to CA-MRSA can be unpredictable, invasive, and more deadly than HAMRSA. As a result, infections with such isolates must be carefully monitored, and the risks due to carriage and transmission of such isolates require further investigation and understanding. The prevalence of MRSA infections in athletes can be much higher than the general population, particularly in athletes in contact sports [30]. However, there are a lack of data regarding the prevalence of MRSA carriage in healthy college athletes, and the molecular characterization of such isolates. Our study sought to address the prevalence and molecular types of MRSA in this population.

It is well established that the anterior nares serves as a reservoir for S. aureus [31-33]. Several studies have examined the colonization rates of both symptomatic and asymptomatic athletes and spectators. The largest group studied has been American football players. Nguyen et al [30] reported an outbreak of CA-MRSA skin and soft tissue infections in 11 players on a college football team. Cultures of the anterior nares only identified 26 of 99 healthy players carrying S. aureus, of which $8 \%$ were 
MRSA. However, only 1 sample was obtained, rather than multiple samples over 12 weeks, as in this study. In our study the overall colonization rate of MRSA in all athletes and their support staff was $36.8 \%$. Begier et al. [34] reported an outbreak of MRSA infection among college football players due to a USA300 clone that was positive for the lukFS-PV genes. Nasal swabs were collected from the athletes and staff, but none were found to carry MRSA. However, 48.5\% were colonized with MSSA. Kazakora et al. [11] reported similar findings in regard to an outbreak of MRSA infections in a professional football team. Nasal swab cultures were collected from all athletes, but again MRSA carriage was not found. [34]. It is likely these participants were carrying MRSA, but in sites other than the anterior nares. Although the number of participants per sport was relatively small, and ethnicity, socio-economic background, or family history of MRSA infection was not noted, this study was distinct in that there had been no reported outbreak of MRSA in the population we examined, and only one active infection with MRSA occurred during the 12-week study. A novel aspect of our study was continuous sample collection from 3 anatomic sites over a 12-week period, as opposed to a one-time nares-only collection, as in previous studies [11,34]. We expected that culturing multiple sites would identify a higher rate of transient MRSA colonization. Transient MRSA isolation was evident in multiple participants throughout the study, either with the same MRSA isolate (e.g. participant \#s 41, 55,57 ) or with varying isolates (e.g. participant \#s 52, 90, 108). However, the profile of persistent or transient carriage of a particular isolate by a participant was incomplete due to a substantial number of participants not being available for culture on any given week.

As reported in previous studies [10,11], athletes in contact sports have higher carriage rates of MRSA. In fact, participants in sports involving greater physical contact, have the most significant carriage rate for MRSA. In our study, of the parameters examined, the sport was the only predictor of MRSA colonization. The highest colonization rate was associated with the contact sport wrestling, followed by baseball. Athletes participating in football were not tested because only athletes participating in Spring sports were involved in this study. However, the sport with the lowest level of colonization was women's basketball, and no participants in women's lacrosse were found to be persistent carriers. Since less contact would be expected in baseball than in basketball or lacrosse (though not to the level of contact in wrestling) gender may also be a factor in CA-MRSA colonization. Nonetheless, these results suggest that athletes who have more physical contact with commonly used fomites or teammates may need to be monitored more closely for signs of potential MRSA infections.
In the United States, MRSA clones USA300, USA400 and often USA700 are the most prevalent and carry the SCCmec IV cassette [23]; USA300 is currently responsible for the majority of morbidity and mortality associated with MRSA, particularly in athletes $[6,10]$. Of the select isolates represented in Table 2, MRSA carriage among the athletes in this study followed the same pattern; all isolates from which a clear SCCmec type could be identified were confirmed to carry a SCCmec IV cassette, which is most commonly found in USA300 and USA400 clones. As expected, USA300/400 clones made up over $69 \%$ of the confirmed type IV isolates tested. Of the 13 participants from whom MRSA isolates were characterized, 6 were colonized with USA300/400 MRSA types. Of interest was that in this population 3 of the participants were colonized with USA700 MRSA isolates and 3 were colonized with USA800 MRSA isolates. Although strains of USA700 commonly occur in the community, USA800 isolates are more often associated with hospital-acquired infections, but community isolates are not uncommon [23]. Furthermore, Stevens et al. reported the presence of USA800 from women's collegiate basketball players [35]. Unfortunately, we did not determine if any of the participants from whom USA800 isolates were recovered had visited a hospital or were in contact with people who had been hospitalized or had visited a hospital. However, one isolate could not be classified into an established USA type by PFGE or PCR, but was determined to be a novel MLST and spa type. This was the only MRSA isolate from this individual, and therefore due to its transient nature its significance cannot be determined. The most common spa type found among these isolates was t008, which represents only $5.99 \%$ of isolates in the Ridom SpaServer database (http://www.spaserver.ridom.de), but in our study type t008 had a much higher frequency $(42.8 \%$ of isolates typed and 3 of 13 participants studied). The t008 type corresponds to the common USA300 clone, and all of the USA300 isolates characterized in this study were spa type t0008, as well as one USA400 type. The two other spa types found in multiple participants were also more common than what is represented in the Ridom database: t002 accounted for $14.2 \%$ of our isolates (6.4\% average) or in 2 of 13 participants studied. The spa type t128 accounted for $19 \%$ of our isolates (0.05\% average) or in 2 of 13 participants studied (http://www.spaserver.ridom.de).

We were also interested in determining if MRSA isolates from healthy participants carried any of the staphylococcal toxin genes, and if so which ones. We detected 9 toxin genes among the 40 MRSA isolates tested. The most common genes found were $l u k F S-P V$, eta, and $e t b$. The lukFS-PV genes encode for the Panton-Valentine leukocidin toxin. MRSA isolates carrying these genes have been shown to be responsible for more severe disease and 
clinical symptoms, including necrotic lesions of the skin [36]. The eta/etb genes encode for exfoliative toxins that may enhance the transmission/progression of a MRSA infection through skin-skin contact, due to the destruction of the epidermal barrier [37]. However, during the course of this study only 1 athlete developed a MRSA infection. Although the presence and expression of toxin genes may potentially make the bacteria more virulent following infection, there was no correlation between the presence of toxin genes and predisposition to infection in these athletes. Therefore, other predisposing conditions may be necessary to initiate infection.

Most participants were not colonized with the same clone continuously throughout the study, nor were they colonized in the same site. While it is quite possible that the presence of MRSA was missed on a particular culture or due to a participant's occasional absence, the variation in toxin genes further suggested that some participants may be prone to carriage or colonization with MRSA, but that residence by individual clones varies. Thus far, studies are lacking that have followed colonization of healthy individuals over time with MRSA to evaluate the persistence of individual strains on a subject over time. Such results have not been commonly reported because most studies have examined MRSA from infected individuals, rather than the prevalence of carriage and colonization in a healthy population. Based on PFGE analysis, it was apparent that strains were transmitted between teammates participating in wrestling and baseball. The mechanism of such transmission was not determined, but is likely through direct contact (particularly in sports such as wrestling) or indirectly through common contact with contaminated fomites (which may occur in locker rooms or handling common pieces of equipment).

\section{Conclusions}

This study indicated that participants in sports are more likely to carry or be colonized with MRSA than the general population, that such carriage may occur in multiple sites other than the nares, and that transient colonization with different strains was common. Precautions to minimize transmission of MRSA between athletes or equipment and facilities used by athletes and staff may be warranted.

\section{Limitations and future work}

A questionnaire was given to each participant asking about residence, previous history of skin or soft tissue infection within 12 months and any previous history of MRSA diagnosis. The specific participants with previous MRSA infection history were not identified to those processing the samples. Therefore, we know only that 1 baseball player and 1 wrestler were previously infected with MRSA. Comparisons between these previously infected individuals and other athletes are lacking. Due to participants scheduling conflicts, twelve weeks of sample collections were not completed on all participants. Future work could include molecular characterization on all 139 MRSA isolates. This could allow for more in-depth comparisons of isolates shared between sports, or comparison of staff versus sport participants.

\section{Abbreviations}

MRSA: Methicillin-resistant Staphylococcus aureus; CA-MRSA: Community-acquired MRSA; HA-MRSA: Hospital-acquired MRSA; PFGE: Pulsed filed gel electrophoresis; PCR: Polymerase chain reaction; MLST: Multi-locus sequence typing; PBP2a: Penicillin binding protein 2a; SCCmec: Staphylococcal cassette chromosome mec; Ccr. Cassette chromosome recombinase; Pvl: Panton-Valentine leukocidin gene; Tst: Toxic shock staphylococcal toxin-1; lukFS-PV: Panton-Valentine leukocidin; PFT: Pulse field type.

\section{Competing interests}

The authors declare that they have no competing interests. Media was provided by BD Diagnostics, Sparks, MD.

\section{Authors' contributions}

AC carried out cultures and molecular typing and wrote the initial draft and reviewed the final draft of the manuscript. TAG conceived the protocol and collected specimens for processing. PGB conceived the protocol and collected specimens for processing. SW performed the statistical analyses. MRP helped conceive the experiments and protocols. TJl conceived the molecular studies, oversaw sample culturing and processing, and wrote the final draft of the manuscript. All authors read and approved the final manuscript.

\section{Acknowledgements}

We would like to thank Elliot Rank and Michele Althouse from BD Biosciences for supplying microbiological reagents, as well as excellent technical advice. We also thank Kristin Knight for technical assistance with PFGE and Troy W. Akers for assistance with specimen collection and processing.

\section{Author details}

${ }^{1}$ Virginia-Maryland Regional College of Veterinary Medicine, Blacksburg, VA, USA. ${ }^{2}$ Edward Via Virginia College of Osteopathic Medicine, Blacksburg, VA 24061, USA. ${ }^{3}$ Virginia Tech Carilion School of Medicine, Virginia Polytechnic Institute and State University, Life Sciences 1, 970 Washington St. SW, Blacksburg, VA 24061, USA.

Received: 8 April 2014 Accepted: 13 July 2014

Published online: 02 August 2014

\section{References}

1. Klevens RM, Edwards JR, Tenover FC, McDonald LC, Horan T, Gaynes R: Changes in the epidemiology of methicillin-resistant Staphylococcus aureus in intensive care units in US hospitals, 1992-2003. Clin Infect Dis 2006, 42(3):389-391.

2. Styers D, Sheehan DJ, Hogan P, Sahm DF: Laboratory-based surveillance of current antimicrobial resistance patterns and trends among Staphylococcus aureus: 2005 status in the United States. Ann Clin Microbiol Antimicrob 2006, 5:2.

3. Salmenlinna S, Lyytikainen O, Vuopio-Varkila J: Community-acquired methicillin-resistant Staphylococcus aureus, Finland. Emerg Infect Dis 2002, 8:602-607.

4. Udo EE, Pearman JW, Grubb WB: Genetic analysis of community isolates of methicillin-resistant Staphylococcus aureus in Western Australia. J Hosp Infect 1993, 25:97-108.

5. Vandenesch F, Naimi T, Enright MC, Lina G, Nimmo GR, Heffernan H, Liassine N, Bes M, Greenland T, Reverdy ME, Etienne J: Community-acquired methicillinresistant Staphylococcus aureus carrying Panton-Valentine leukocidin genes: worldwide emergence. Emerg Infect Dis 2003, 9:978-984. 
6. Said-Salim B, Mathema B, Kreiswirth BN: Community-acquired methicillinresistant Staphylococcus aureus: an emerging pathogen. Infect Control Hosp Epidemiol 2003, 24:451-455.

7. David MZ, Daum RS: Community-associated methicillin-resistant Staphylococcus aureus: epidemiology and clinical consequences of an emerging epidemic. Clin Microbiol Rev 2010, 23(3):616-687.

8. Diep BA, Gill SR, Chang RF, Phan TH, Chen JH, Davidson MG, Lin F, Lin J, Carleton HA, Mongodin EF, Sensabaugh GF, Perdreau-Remington F: Complete genome sequence of USA300, an epidemic clone of community-acquired meticillin-resistant Staphylococcus aureus. Lancet 2006, 367:731-739.

9. John CC, Schreiber JR: Therapies and vaccines for emerging bacterial infections: learning from methicillin-resistant Staphylococcus aureus. Pediatr Clin North Am 2006, 53:699-713.

10. Cohen PR: The skin in the gym: a comprehensive review of the cutaneous manifestations of community-acquired methicillin-resistant Staphylococcus aureus infection in athletes. Clin Dermatol 2008, 26:16-26.

11. Kazakova SV, Hageman JC, Matava M, Srinivasan A, Phelan L, Garfinkel B, Boo T, McAllister S, Anderson J, Jensen B, Dodson D, Lonsway D, McDougal LK, Arduino M, Fraser VJ, Killgore G, Tenover FC, Cody S, Jernigan DB: A clone of methicillin-resistant Staphylococcus aureus among professional football players. N Engl J Med 2005, 352:468-475.

12. Lemaire S, Fuda C, Van Bambeke F, Tulkens PM, Mobashery S: Restoration of susceptibility of methicillin-resistant Staphylococcus aureus to betalactam antibiotics by acidic pH: role of penicillin-binding protein PBP 2a. J Biol Chem 2008, 283:12769-12776.

13. Chongtrakool P, Ito T, Ma XX, Kondo Y, Trakulsomboon S, Tiensasitorn C, Jamklang M, Chavalit T, Song JH, Hiramatsu K: Staphylococcal cassette chromosome mec (SCCmec) typing of methicillin-resistant Staphylococcus aureus strains isolated in 11 Asian countries: a proposal for a new nomenclature for SCCmec elements. Antimicrob Agents Chemother 2006, 50:1001-1012

14. International Working Group on the Staphylococcal Cassette Chromosome elements: Currently identified SCCmec types in S. aureus strains. 2012. http:// www.sccmec.org/Pages/SCC_TypesEN.html. Accessed 26 Oct 2012.

15. Ito T, Ma XX, Takeuchi F, Okuma K, Yuzawa H, Hiramatsu K: Novel type V staphylococcal cassette chromosome mec driven by a novel cassette chromosome recombinase, ccrC. Antimicrob Agents Chemother 2004 , 48:2637-2651

16. Okuma K, Iwakawa K, Turnidge JD, Grubb WB, Bell JM, O'Brien FG, Coombs GW, Pearman JW, Tenover FC, Kapi M, Tiensasitorn C, Ito T, Hiramatsu K: Dissemination of new methicillin-resistant Staphylococcus aureus clones in the community. J Clin Microbiol 2002, 40:4289-4294.

17. Mongkolrattanothai K, Boyle S, Kahana MD, Daum RS: Severe Staphylococcus aureus infections caused by clonally related communityacquired methicillin-susceptible and methicillin-resistant isolates. Clin Infect Dis 2003, 37:1050-1058.

18. Naimi TS, LeDell KH, Como-Sabetti K, Borchardt SM, Boxrud DJ, Etienne J, Johnson SK, Vandenesch F, Fridkin S, O'Boyle C, Danila RN, Lynfield R: Comparison of community- and health care-associated methicillin-resistant Staphylococcus aureus infection. J Amer Med Assn 2003, 290:2976-2984.

19. Francis JS, Doherty MC, Lopatin U, Johnston CP, Sinha G, Ross T, Cai M, Hansel NN, Perl T, Ticehurst JR, Carroll K, Thomas DL, Nuermberger E, Bartlett JG: Severe community-onset pneumonia in healthy adults caused by methicillin-resistant Staphylococcus aureus carrying the PantonValentine leukocidin genes. Clin Infect Dis 2005, 40:100-107.

20. Chambers HF: Community-associated MRSA-resistance and virulence converge. N Engl J Med 2005, 352:1485-1487.

21. Safdar N, Narans L, Gordon B, Maki DG: Comparison of culture screening methods for detection of nasal carriage of methicillin-resistant Staphylococcus aureus: a prospective study comparing 32 methods. J Clin Microbiol 2003, 41:3163-3166.

22. Nouwen JL, Ott A, Kluytmans-Vandenbergh MF, Boelens HA, Hofman A, van Belkum A, Verbrugh HA: Predicting the Staphylococcus aureus nasal carrier state: derivation and validation of a "culture rule". Clin Infect Dis 2004, 39:806-811.

23. McDougal LK, Steward CD, Killgore GE, Chaitram JM, McAllister SK, Tenover FC: Pulsed-field gel electrophoresis typing of oxacillin-resistant Staphylococcus aureus isolates from the United States: establishing a national database. J Clin Microbiol 2003, 41:5113-5120.
24. Palavecino E: Clinical, epidemiological, and laboratory aspects of methicillin-resistant Staphylococcus aureus (MRSA) Infections. Methods Mol Biol 2014, 1085:1-24.

25. Zhang K, McClure JA, Elsayed S, Louie T, Conly JM: Novel multiplex PCR assay for characterization and concomitant subtyping of staphylococcal cassette chromosome mec types I to $\mathrm{V}$ in methicillin-resistant Staphylococcus aureus. J Clin Microbiol 2005, 43:5026-5033.

26. Mehrotra M, Wang G, Johnson WM: Multiplex PCR for detection of genes for Staphylococcus aureus enterotoxins, exfoliative toxins, toxic shock syndrome toxin 1, and methicillin resistance. J Clin Microbiol 2000, 38:1032-1035

27. Enright MC, Day NP, Davies CE, Peacock SJ, Spratt BG: Multilocus sequence typing for characterization of methicillin-resistant and methicillin-susceptible clones of Staphylococcus aureus. J Clin Microbiol 2000, 38:1008-1015.

28. Harmsen D, Claus H, Witte W, Rothganger J, Turnwald D, Vogel U: Typing of methicillin-resistant Staphylococcus aureus in a university hospital setting by using novel software for spa repeat determination and database management. J Clin Microbiol 2003, 41:5442-5448.

29. Zong Z, Peng C, Lu X: Diversity of SCCmec elements in methicillinresistant coagulase-negative staphylococci clinical isolates. PLoS One 2011, 6:e20191.

30. Nguyen DM, Mascola L, Brancoft E: Recurring methicillin-resistant Staphylococcus aureus infections in a football team. Emerg Infect Dis 2005, 11:526-532.

31. Borchardt SM, Yoder JS, Dworkin MS: Is the recent emergence of community-associated methicillin-resistant Staphylococcus aureus among participants in competitive sports limited to participants? Clin Infect Dis 2005, 40:906-907.

32. Miller M, Cespedes C, Bhat M, Vavagiakis P, Klein RS, Lowy FD: Incidence and persistence of Staphylococcus aureus nasal colonization in a community sample of HIV-infected and -uninfected drug users. Clin Infect Dis 2007, 45:343-346.

33. Wertheim HF, Melles DC, Vos MC, van Leeuwen W, van Belkum A, Verbrugh HA, Nouwen JL: The role of nasal carriage in Staphylococcus aureus infections. Lancet Infect Dis 2005, 5:751-762

34. Begier EM, Frenette K, Barrett NL, Mshar P, Petit S, Boxrud DJ, Watkins-Colwell K, Wheeler S, Cebelinski EA, Glennen A, Nguyen D, Hadler JL: A high-morbidity outbreak of methicillin-resistant Staphylococcus aureus among players on a college football team, facilitated by cosmetic body shaving and turf burns. Clin Infect Dis 2004, 39:1446-1453.

35. Whitman TJ, Schlett CD, Grandits GA, Millar EV, Mende K, Hospenthal DR, Murray PR, Tribble DR: Chlorhexidine gluconate reduces transmission of methicillin-resistant Staphylococcus aureus USA300 among Marine recruits. Infect Control Hosp Epidemiol 2012, 33:809-816.

36. Otto M: Basis of virulence in community-associated methicillin-resistant Staphylococcus aureus. Annu Rev Microbiol 2010, 64:143-162.

37. Bukowski M, Wladyka B, Dubin G: Exfoliative toxins of Staphylococcus aureus. Toxins 2010, 2:1148-1165.

doi:10.1186/s12941-014-0033-5

Cite this article as: Champion et al:: Prevalence and characterization of methicillin-resistant Staphylococcus aureus isolates from healthy university student athletes. Annals of Clinical Microbiology and Antimicrobials 2014 13:33.

\section{Submit your next manuscript to BioMed Central and take full advantage of:}

- Convenient online submission

- Thorough peer review

- No space constraints or color figure charges

- Immediate publication on acceptance

- Inclusion in PubMed, CAS, Scopus and Google Scholar

- Research which is freely available for redistribution 